\title{
Experienced consent in geriatrics research: a new method to optimize the capacity to consent in frail elderly subjects
}

Marcel G M Olde Rikkert, John H L van den Bercken, Henk A M J ten Have and Willibrord H L Hoefnagels

\author{
University of Nijmegen, the Netherlands
}

\begin{abstract}
Objectives - Cognitive and sensory difficulties frequently jeopardize informed consent of frail elderly patients This study is the first to test whether preliminary research experience could enhance geriatric patients' capacity to consent.

Design/setting - $A$ step-wise consent procedure was introduced in a study on fluid balance in geriatric patients. Eligible patients providing verbal consent participated in a try-out of a week, during which bioelectrical impedance and weight measurements were performed daily. Afterwards, written informed consent was requested. Comprehension, risk and inconvenience scores (ranges: 0-10) were obtained before and after the try-out by asking ten questions about the study's essentials and by asking for a risk and inconvenience assessment on a ten-points rating scale. Subjects and results - Seventy of the 78 eligible subjects started the try-out and 53 (68\%) provided written consent. The comprehension score increased from $5 \cdot 0$ $( \pm 2 \cdot 3)$ to $7 \cdot 0( \pm 1 \cdot 9)$ following the try-out $(P<0.001)$. The number of subjects capable of weighing risks and inconveniences increased from 32 to $48(P<0.001)$.

Conclusions - Research experience improved the capacity to consent, still enabling an acceptable participation rate. Therefore, experienced consent seems a promising tool to optimize informed consent in frail elderly subjects.
\end{abstract}

\section{Introduction}

To obtain informed consent properly requires a great effort on the part of researchers attempting to study frail elderly patients. First, the researcher must develop a procedure to assess which patients are capable of providing informed consent. Depending on the type of study, patients incapable of consenting must be excluded or consent by proxy must be obtained. Much time, energy and creativity must be invested in informing subjects appropriately to allow

\section{Key terms}

Medical ethics; informed consent; geriatrics; human experimentation; mental competency. for free and autonomous decisions. Ultimately, informed consent has to be obtained from a sufficient number of subjects to meet sample size requirements. To explain highly sophisticated research techniques, commonly used in current medical research, to geriatric patients, born in an era in which medicine was mostly "handmade" may be a formidable task. ${ }^{12}$ The high prevalence of cognitive, visual, auditory and language deficits in this population may constrain acquiring, understanding and adequately weighing the information provided by the investigator. Helping the subject to understand as fully as possible, and ascer-? taining how well the subject understands, is often necessary in geriatric research.

Important conditions for reaching these goals are: adequate assessment of capacity to consent, ${ }^{34}$ allowing extensive time for the consent process, ${ }^{2}$ and matching consent material in format and presentation to the cognitive, visual and hearing capacities of frail elderly patients. ${ }^{5}$ So far, most authors have focused on all kinds of visual and hearing aids, such as pictures, vignettes, storybooks and audio- or videotapes. ${ }^{6} 7$ However, Tymchuk showed that these aids proved a distraction rather than an aid for elderly subjects. ${ }^{8}$ Educational training was also suggested as a method of enhancing decision-making capacity. ${ }^{9}{ }^{10}$ As far as we know, this is the first study aimed at determining the effects of research experience on the capacity to consent.

\section{Patients and methods}

This study was part of a non-therapeutic research project aimed at the validation of multifrequency bioelectrical impedance analysis (MFBIA) in monitoring fluid balance in geriatric patients. Multifrequency bioelectrical impedance analysis has been validated already in healthy elderly subjects ${ }^{11}$ and it promised to be a quick and non-invasive method also suitable for frail elderly patients. A three-part consent procedure was applied: 
to December 31, 1995 to the Department of Geriatric Medicine of the University Hospital Nijmegen were screened for their eligibility by a trainee in geriatric medicine and the supervising geriatrician. Neither were involved in other parts of this study. Patients are admitted to our department on an needs-based policy, which means that we only admit frail elderly patients, suffering from multiple medical or psychiatric diseases at the same time, in whom the precious balance that characterizes their physical, psychological and social functioning, is disturbed. In total, 140 patients (64\%) had to be excluded because of: (a) having a pacemaker $(n=6)$; (b) severe or terminal illnesses $(n=6)$; (c) psychogeriatric diseases likely to interfere with research compliance $(n=9)$; (d) moderate or severe dementia according to the Clinical Dementia Rating scale (CDR $>1 ; n=89$ ) (12). This screening was carried out during the first days of admission. Another 30 subjects had to be excluded because of logistic limitations which meant that a maximum of six patients could participate in the study at any one time.

2. Research experience was provided by means of a try-out period of a week. Verbal and written information concerning the try-out was given to all eligible subjects $(n=78)$. The study's essentials were explained, tailored as much as possible to the subjects' hearing and visual capacities. In $40 \%$ of the potential subjects family members were willing to accompany them while they were informed. Seventy subjects $(90 \%)$ provided verbal consent to participate in the try-out, during which MFBIA and weight measurements were carried out daily. Multifrequency bioelectrical impedance analysis was performed each morning just following a subject's awakening while still lying supine. The measurements took fifteen minutes using four adhesive electrodes placed on hand and foot. ${ }^{13}$ Try-out measurements were performed in exactly the same way as planned in the MFBIA validation project and did not interfere with necessary clinical investigations or treatments.

Subjects were characterized on the basis of answers to questions about their educational level and way of living before hospitalization. Cognition, mobility, performances in activities of daily living (ADL) and life satisfaction were also determined by measuring four generally recommended geriatric assessment scores: Mini-Mental State Examination (MMSE); Barthel-index; Tinetti Balance and Gait Evaluation-score, and Philadelphia Geriatric Center Morale Scale (PGCMS)..$^{1415}$

3. Following the try-out written informed consent was requested. Beforehand, the same verbal information had been given once more. This time $45 \%$ of the subjects were accompanied by family members. Following written consent daily measurements were continued until discharge, unless subjects withdrew their consent. The study could also be stopped by the responsible geriatrician in subjects with a severely worsening clinical condition. After discharge it was determined that 19 of the 70 participating subjects $\overrightarrow{\bar{\sigma}}$. $(27 \%)$ were diagnosed by the responsible geriatrician? as suffering from a mild degree of dementia according $\overrightarrow{\vec{F}}$ to DSM IIIR criteria. In addition, ten of the non-? demented subjects, and four of the demented subjects? were diagnosed as suffering from a major depression, $\frac{\bar{c}}{\bar{c}}$ also assessed using DSM IIIR criteria.

\section{CAPACITY TO CONSENT}

Assessment of the capacity to consent was carried out before and after experiencing research by testing comprehension and ability to weigh risks and incon- $\vec{\omega}$ veniences. Comprehension of research information was tested asking ten multiple-choice questions that $\stackrel{\nabla}{\mathrm{d}}$ covered six basic categories of relevant information: ${ }_{\omega}^{N}$ benefits, departure from ordinary medical practice, or risks, inconveniences and tasks, purposes, and the subjects' rights (see appendix). Assigning one point to each properly answered question resulted in $a$ 음 comprehension score ranging from zero to ten. This comprehension assessment was adapted from the "two-part consent form" suggested by Miller. ${ }^{16} \frac{\overrightarrow{0}}{\circ}$ Additionally, the ability to weigh research informa- $\stackrel{\mathbb{D}}{7}$ tion was tested. To this end, subjects were asked to $\vec{\oplus}$ assign a number, ranging from zero to ten, to expected risks and inconveniences. "Zero" mea. that a subject expected no risks or inconveniences; "ten" meant that the study was considered to be vey risky or associated with substantial inconvenience. Comprehension, risk and inconvenience scores were $\stackrel{0}{\circ}$ determined without presence of family members. $\stackrel{\mathbb{Q}}{\Omega}$ Each time a nurse was present as independent $\overrightarrow{\overrightarrow{0}}$ "consent auditor", who could answer the patient's 3 questions raised after the consent session. All measurements were carried out by the same? researcher (MOR), only for the Barthel-indexes were nursing observations also used.

\section{ETHICS}

The research protocol for the MFBIA project, $\frac{\delta}{3}$ including this study of experienced consent, was approved of by the local committee on human experimentation. Only subjects judged as capable of $\frac{}{O}$ giving informed consent were allowed to be included in this non-therapeutic study. The committee agreed $N$ that this precondition was translated into practice by $\mathrm{N}$ excluding all subjects assessed as moderately or N severely demented $(C D R>1)$.

\section{STATISTICAL ANALYSIS}

The effect of research experience on the comprehension score was tested by means of paired t-tests. The try-out effect on the number of subjects capable of $\frac{T}{0}$ weighing expected risks and inconvenience was $\stackrel{\mathrm{D}}{\mathrm{D}}$

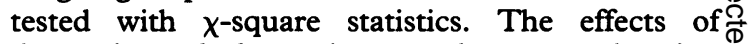

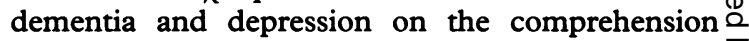
score was tested with two-way analysis of variance (ANOVA) using average and difference scores of the measurements before and after research experience. 
Table 1 Means and standard deviations of comprehension, risk and inconvenience scores before and after research experience for all subjects and for mildly demented and non-demented subjects separately

\begin{tabular}{|c|c|c|c|c|}
\hline & \multicolumn{4}{|c|}{ All subjects } \\
\hline & Before (SD) & $N^{\star}$ & After (SD) & $N^{*}$ \\
\hline \multirow[t]{2}{*}{$\begin{array}{l}\text { Comprehension score } \\
\text { Risk score } \\
\text { Inconvenience score }\end{array}$} & $\begin{array}{l}5 \cdot 0(2 \cdot 3) \\
2 \cdot 5(2 \cdot 7) \\
4 \cdot 6(2 \cdot 5)\end{array}$ & $\begin{array}{l}53 \\
33 \\
40\end{array}$ & $\begin{array}{l}7 \cdot 0(1 \cdot 9) \dagger \\
1 \cdot 3(2 \cdot 5) \\
3 \cdot 2(2 \cdot 3)\end{array}$ & $\begin{array}{l}53 \\
48 \\
50\end{array}$ \\
\hline & \multicolumn{4}{|c|}{ Mildly demented subjects } \\
\hline \multirow[t]{2}{*}{$\begin{array}{l}\text { Comprehension score } \\
\text { Risk-score } \\
\text { Inconvenience score }\end{array}$} & $\begin{array}{l}3 \cdot 5(1 \cdot 4) \\
5 \cdot 0(3 \cdot 1) \\
5 \cdot 2(2 \cdot 0)\end{array}$ & $\begin{array}{l}16 \\
8 \\
9\end{array}$ & $\begin{array}{l}5 \cdot 8(1 \cdot 9) \dagger \\
1.8(2 \cdot 0) \\
3.4(2 \cdot 4)\end{array}$ & $\begin{array}{l}16 \\
14 \\
14\end{array}$ \\
\hline & \multicolumn{4}{|c|}{ Non-demented subjects } \\
\hline $\begin{array}{l}\text { Comprehension score } \\
\text { Risk-score } \\
\text { Inconvenience score }\end{array}$ & $\begin{array}{l}5 \cdot 6(2 \cdot 3) \\
1 \cdot 8(2 \cdot 1) \\
4 \cdot 4(3 \cdot 6)\end{array}$ & $\begin{array}{l}37 \\
25 \\
31\end{array}$ & $\begin{array}{l}7 \cdot 5(1 \cdot 7) \dagger \\
1 \cdot 2(2 \cdot 1) \\
3 \cdot 7(1 \cdot 5)\end{array}$ & $\begin{array}{l}37 \\
34 \\
36\end{array}$ \\
\hline
\end{tabular}

$\star$ Not all subjects were able to weigh expected risks and inconveniences.

$\dagger \mathrm{P}<0 \cdot 001$, by t-test.

Differences in sex, social characteristics and in the prevalence of dementia and depression between subjects providing and subjects refusing written consent were compared using $\chi$-square tests. Initial comprehension scores and geriatric assessment scores in these two groups were compared with t-tests. Standard deviations (SD) are presented within parentheses, unless otherwise indicated.

\section{Results}

The try-out effect on the comprehension scores could be tested in 53 subjects ( 17 men, 36 women) who provided written informed consent. Mean age of the participants was $80 \cdot 1$ years (range: $70-92$ ). Initially the subjects answered only $5 \cdot 0(2 \cdot 3)$ of the ten questions correctly (table 1 ). This number increased to $7 \cdot 0$ (1.9) after the try-out. The mean increase in comprehension score was highly significant with a $95 \%$ confidence interval ranging from 1.5 to $2.7(\mathrm{P}<0.001)$. The first risk assessment could not be completed by 20 subjects. This number declined to five at the assessment following research experience. Similarly, the number of subjects that could not answer the inconvenience question declined from 13 to three following the run-in. The total number of subjects that could weigh both risks and inconveniences increased significantly from 32 to $48 \quad(P<0.001)$. The 21 subjects who were unable to perform these assessments initially, had a lower comprehension score before the try-out than the 32 who could weigh risks and inconveniences: $4.0(2.2)$ v $5.6(2.2)(P<0.05)$. However, the beneficial effect of the try-out on the comprehension scores was similar in both groups.

In the 53 subjects sub-group analyses were performed on the 16 subjects diagnosed as having a mild degree of dementia (table 1), and the 11 subjects suffering from a major depression. Mildly demented subjects had a lower average level of the comprehension score than non-demented subjects
$(P<0.05)$. However, the try-out effect on the comprehension score was similar in both groups. Suffering from a depression had no effect on the initial level of the comprehension score, nor on the try-out effect (results not shown). There were no differences in the prevalence of dementia and depression between the 32 subjects who could, and the 21 . who could not, weigh risks and inconveniences.

EFFECT OF THE TRY-OUT ON PARTICIPATION RATES This step-wise consent procedure resulted in a participation-rate of $68 \%(53 / 78)$ of all eligible subjects. During the try-out seven subjects withdrew consent. Ten subjects refused informed consent to continue research following the try-out. Three of them withdrew consent because they felt electric shocks during MFBIA, although no muscle twitches could be observed. Seven of the 53 participating subjects stopped their participation before discharge. Another seven subjects who became delirious during admission were withdrawn from the study. However, MFBIA data from all 53 subjects could be analyzed because they participated long enough in the study (mean participation: 29 days; range: 8-143 days). The characteristics of the 53 subjects who provided written consent were compared with those of the 17 subjects who refused further participation. More subjects in the group providing written consent only completed primary school $(n=29)$ than in the group refusing written consent $(n=2$, $P<0.01)$. There were no differences in age or sex distribution and similar numbers of subjects lived independently $(33 / 53 \mathrm{v} 10 / 17)$ before admission. Refusing consent was characterized by having higher initial inconvenience scores $(P<0.05$, table 2$)$. There was no significant difference in geriatric assessment-scores between the participating and the non-participating subjects and there were also no differences in the prevalences of dementia and depression. As shown by substantial standard devia- 
Table 2 Comparison of initial comprehension, risk and inconvenience scores, and geriatric assessment scores between subjects providing and subjects refusing written informed consent

\begin{tabular}{|c|c|c|c|c|}
\hline & Informed consent & $N^{*}$ & No informed consent & $N^{*}$ \\
\hline $\begin{array}{l}\text { Comprehension score } \\
\text { Risk score } \\
\text { Inconvenience score }\end{array}$ & $\begin{array}{l}5 \cdot 0(2 \cdot 3) \\
2 \cdot 5(2 \cdot 7) \\
4 \cdot 6(2 \cdot 5) \dagger\end{array}$ & $\begin{array}{l}53 \\
33 \\
40\end{array}$ & $\begin{array}{l}6.2(2.9) \\
3.6(0.8) \\
6.5(1.3)\end{array}$ & $\begin{array}{l}17 \\
14 \\
14\end{array}$ \\
\hline $\begin{array}{l}\text { MMSE }(0-30 \\
\text { PGCMS }(0-17) \\
\text { Tinetti-index }(0-28) \\
\text { Barthel-index }(0-20)\end{array}$ & $\begin{array}{l}22 \cdot 9(5 \cdot 1) \\
10 \cdot 3(4 \cdot 7) \\
15 \cdot 3(9 \cdot 1) \\
13 \cdot 1(5 \cdot 2)\end{array}$ & $\begin{array}{l}53 \\
53 \\
53 \\
53\end{array}$ & $\begin{array}{r}24.9(4 \cdot 1) \\
8 \cdot 7(4 \cdot 5) \\
10 \cdot 5(8 \cdot 6) \\
10 \cdot 6(5 \cdot 4)\end{array}$ & $\begin{array}{l}17 \\
17 \\
17 \\
17\end{array}$ \\
\hline
\end{tabular}

$\star$ Not all subjects were able to weigh expected risks and inconveniences. $+\mathrm{P}<0.05$.

tions on all geriatric assessment scores, there was a considerable heterogeneity in functional performance in both groups.

\section{Discussion}

The process of informed consent is a topic of ethical debate because there may be a conflict of interests between researchers and eligible subjects. International rules, such as the Declaration of Helsinki (last revision, 1989), the Nuremberg Code (1947) and the International Covenant on Civil and Political Rights of the United Nations (1966), require free and informed consent of research subjects. Recently, much has been written about informed consent procedures for medical research, but empirical data on how this complicated process might be optimized in research on elderly subjects are scarce. We will subsequently discuss the results of providing research experience during the informed consent procedure both from the point of view of the potential subjects and the investigator.

There are three major advantages of this step-wise consent procedure from the subjects' point of view. Firstly, research experience seems to improve the capacity to consent in demented and depressed subjects as well as in subjects without psychogeriatric illnesses. This is important because acquisition and understanding of research information may often be limited, as it was initially in this study. ${ }^{17}$ The capacity to consent improved in understanding of the key points of the study, but also in ability to weigh risks and inconveniences. The statistically significant increase in comprehension scores $(+2 \cdot 0)$ was sufficiently large to be considered clinically and ethically significant, because it suggests that subjects were able to understand at least one more of the six basic categories of relevant information mentioned by Miller (ie benefits, departure from ordinary medical practice, risks, inconveniences and tasks, purposes, and the subjects' rights). ${ }^{16}$ Truly informed consent can only be reached if a potential subject is capable of communicating, understanding information and weighing research benefits, risks and inconveniences with regard to the subject's own set of values and goals. ${ }^{8918}$ Therefore, the increase in the number of subjects $(+16)$ who could evaluate risks $\underset{\omega}{\tilde{\omega}}$ and inconveniences must be considered as a clini- $v$ cally relevant improvement of the capacity to $N$ consent.

Secondly, only by providing research experience may an investigator enable a true risk and inconvenience assessment. Research often seems trivial to investigators in terms of risks and discomfort, but may not seem so trivial to the vulnerable and often frightened elderly population. ${ }^{19}$ In this study mean risk and inconvenience scores decreased following the try-out. However, research experience caused seventeen subjects to stop participating. Three somewhat anxious subjects stopped because they thought that MFBIA caused electric shocks, although it is generally agreed that the small electrical currents involved are below the threshold of perception. ${ }^{20}$ Subjects refusing written consent also had higher inconvenience scores.

Thirdly, by officially scheduling two appointments to discuss participation in the study, and by waiting at least a week before asking for written consent, potential subjects were offered more control about their decision to participate. Though in every study a subject is free to withdraw consent at any moment, it may be easier not to sign a consent form than to withdraw permission after having given written consent. In this study it resulted in the refusal to participate of ten subjects during an evaluative talk about their research experiences.

The researcher, on the other hand, is mainly interested in an informed consent procedure that results in the inclusion of a sufficient number of eligible subjects who can complete the research protocol. Subjects should also understand what is being asked for in the study in order to prevent a high non-compliance rate. The final participation-rate in this study $(68 \%)$ was similar to percentages reported in the three published studies reporting explicitly on the consent process and the consent rate in research on frail elderly subjects. ${ }^{1221}$ Multifrequency bioelectrical impedance analysis, requiring that subjects stay quietly supine for fifteen minutes, could be performed on all subjects. Subjects who were too restless or agitated because of psychosis, dementia or 
delirium were excluded in the first stage of the consent procedure or were withdrawn from the study, because they intercurrently developed a delirium. The compliance during the study was high, even though seven subjects stopped participating before discharge. Their withdrawal might be explained by the fact that hospitalization lasted longer than expected beforehand: mean duration was 37.0 days with a range of 12 to 145 days. Daily MFBIA may become quite burdensome after a while, although it is a quick and non-invasive procedure. In conclusion, providing research experience before asking for written consent also fulfilled the researcher's needs by enabling the inclusion of a sufficient number of subjects capable of understanding the essentials of the study without having to spend too much time, energy or financial resources.

From the investigator's point of view, applying a try-out period in research on elderly subjects to optimize the subjects' compliance has been promoted before. ${ }^{22} \mathrm{~A}$ try-out used for this purpose is generally called a run-in period. Introducing a run-in period was described as complicated and only worthwile when aimed at excluding poor compliers. ${ }^{23}$ There are important differences between a try-out as used in this study and a run-in to enhance compliance. First, written informed consent was asked following the try-out but generally this precedes a run-in. Secondly, the try-out was developed to benefit the potential subjects, while the run-in was developed to benefit the investigator. We could not find any study describing the beneficial effects of a try-out on elderly subjects' capacity to consent. Tymchuk suggested that educational training might improve the process of informed consent in the elderly, while other methods of improving the capacity to consent failed. ${ }^{58}$ Empirically, he showed the beneficial effects of such a training in mentally retarded mothers, ${ }^{24}$ but not in elderly subjects.

In conclusion, research experience provided in a run-in may improve the quality of the informed consent procedure in geriatrics research. This beneficial effect will vary with the nature and complexity of the study, with the quality of the initial instructions, and with the wordings of questionnaires used to assess understanding of these instructions. In a randomized controlled trial a try-out period could be implemented as an open run-in period, in which all potential subjects receive the experimental treatment. However, it may not always be possible to perform a try-out, as, for example, in a study of invasive diagnostic procedures. In these cases a dummy demonstration of the research measurements may be applicable. Consent obtained following a try-out or such a demonstration could be called "experienced consent" to discriminate it clearly from common informed consent procedures. To justify the implementation of a try-out based on verbal consent, data obtained in the try-out period should not be part of the data set required for the completion of the clinical trial. Try-out data from subjects who provided written consent may be used supplementary to the data set required in the trial, however, data from subjects who did not provide written consent should be deleted. More data, preferably obtained by means of a randomized clinical trial, are necessary to confirm the impression that experienced consent might be a substantial improvement in tailoring research designs to the needs of frail elderly patients.

\section{Acknowledgements}

We are grateful to Jerry $\mathrm{H}$ Gurwitz, MD from the Division of Aging of Harvard Medical School, Brigham and Women's Hospital, Boston USA, and to Martin A van ' $t$ Hof, Statistician from the Department of Methodology, Statistics and Medicine, University of Nijmegen, the Netherlands, for their comments on an earlier version of the manuscript. And we are grateful for the support of The Netherlands Programme for Research on Aging (NESTOR), funded by the Ministry of Education Culture ans Sciences, and the Ministry of Health Welfare and Sports, for this work.

Marcel G M Olde Rikkert, MD, is Research Fellow in Geriatric Medicine, and Willibrord H L Hoefnagels, $M D, P h D$, is Professor of Geriatric Medicine, in the Department of Geriatric Medicine, University Hospital Nijmegen, The Netherlands. Fohn $H L$ van den Bercken is Associate Professor of Diagnostic Decision Making, in the Nijmegen Institute of Cognition and Information, Faculty of Social Sciences of the University of Nijmegen. Henk $A M \mathcal{F}$ ten Have, $M D, P h D$, is Professor of Medical Ethics in the Department of Ethics, Philosophy and History of Medicine, School of Medical Sciences, University of Nijmegen, the Netherlands.

\section{References}

1 Cohen-Mansfield J, Kerin P, Pawlson G, Lipson S, Holdridge $K$. Informed consent for research in a nursing home: processes and issues. The Gerontologist 1988; 28: 355-9.

2 Duffy LB, Wyble SJ, Wilson B, Miles SH. Obtaining geriatric patient consent. Fournal of Gerontological Nursing 1989; 15: 21-4.

3 Berkowitz S. Informed consent, research, and the elderly. The Gerontologist 1978; 18: 237-43.

4 Cassel CK. Ethical issues in the conduct of research in long term care. The Gerontologist 1988; 28: 90-6.

5 Tymchuk AJ, Ouslander JG. Optimizing the informed consent process with elderly people. Educational Gerontology 1990; 16: 245-57.

6 Sachs GA, Cassel CK. Biomedical research involving older human subjects. Law, Medicine $\mathcal{E}$ Health Care 1990; 18: 234-43.

7 Lo B. Assessing decision-making capacity. Law, Medicine E Health Care 1990; 18: 193-201. 
8 Tymchuk JG, Ouslander JG, Rader N. Informing the elderly: a comparison of four methods. Fournal of the American Geriatrics Society 1986; 34: 818-22.

9 High DM. Research with Alzheimer's disease subjects: informed consent and proxy decision making. Fournal of the American Geriatrics Society 1992; 40: 950-7.

10 Tymchuk AJ. An alternative conceptualization of informed consent with people who are elderly. Educational Gerontololgy 1992; 18: 135-47.

11 Visser M, Deurenberg P, Staveren WA van. Multifrequency bioelectrical impedance for assessing total body water and extracellular water in elderly subjects. European fournal of Clinical Nutrition 1995; 49: 256-66.

12 Berg L. Clinical dementia rating. Psychopharmacological Bulletin 1988; 24: 637-9.

13 Lukaski HC, Johnson PE, Bolonchuk WW, Lykken GI. Assessment of fat-free mass using bioelectrical impedance measurements of the human body. American fournal of Clinical Nutrition 1985; 41: 810-7.

14 Fleming KC, Evans JM, Weber DC, Chutka DS. Practical functional assessment of elderly persons: a primary-care approach. Mayo Clinics Proceedings 1995; 70: $890-910$.

15 Joint workshop of the Research Unit of the Royal College of Physicians and the British Geriatrics Society. Standardised assessment scales for elderly people. London: BGS, 1992.
16 Miller R. The two-part consent form. New England fournal of Medicine 1974; 289: 964-6.

17 Lynöe N, Sandlund M, Dahlqvist G, Jacobson L. Informed consent: study of quality of information given to participants in a clinical trial. British Medical fournal 1991; 303: 610-3.

18 Stanley B, Guido J, Stanley M, Shortell D. The elderly patient and informed consent. fournal of the American Medical Association 1984; 252: 1302-6.

19 Annas GJ, Glantz LH. Rules for research in nursing homes. New England fournal of Medicine 1986; 315: 1157-8.

$20 \mathrm{NIH}$ Technology assessment statement. Bioelectrical impedance analysis in body composition measurement. American fournal of Clinical Nutrition 1996; 64: 524S-32S.

21 Brod MS, Feinbloom RI. Feasibility and efficacy of verbal consents. Research on Aging 1990; 12: 364-72. N

22 Ouslander JG, Schnelle JF. Research in nursing homes: or practical aspects. Fournal of the American Geriatrics iv Society 1993; 41: 182-7.

23 Lang JM. The use of a run-in to enhance compliance. Statistics in Medicine 1990; 9: 87-95.

24 Tymchuk AJ, Andron L, Rahbar B. Effective decisionmaking/problem solving training with mentally retarded mothers. American fournal of Mental Retardation 1988; 92: 510-6.

\section{Appendix}

Ten multiple-choice questions asked of geriatric patients to assess their comprehension of relevant research information in a researe project aimed at the validation of bioelectrical impedance measurements in monitoring fluid balance

Questions Possible answers $\quad$ P

1. What is the purpose of this study?

2. Who decides whether you will participate in this study?

3. What is the topic of the study?

4. How will the most important measurement of this study be carried out?

5. How many physicians will carry out these measurements?

6. How often will the measurements take place?

7. How long will the study last?

8. What effects can the electrical current have?

1a. Optimizing your treatment.

1b. Optimizing your investigation.

1c. Optimizing the research instrument.

1d. I don't know.

2a. I do.

2b. My family.

2c. The physician

2d. I don't know.

3a. Sleep disturbances.

3b. Fluid balance.

3c. Dizziness

3d. I don't know.

4a. With X-rays

4b. With electrical current

4c. By blood investigations

4d. I don't know.

5a. One physician

5b. Two physicians

5c. Three physicians

5d. I don't know.

6a. Twice a day.

6b. Twice a week.

6c. Once a day.

6d. I don't know.

7a. One week

7b. Two weeks

7c. Until discharge

7d. I don't know.

8a. You cannot feel it.

8 b. It can give shocks.

$8 \mathrm{c}$. It can be very risky.

$8 d$. I don't know.

9a. The physician

9b. I can.

9c. Both me and my physician

9d. I don't know.

10. Who will possibly benefit from the study?

10b. Future patients.

10c. Both me and future patients

$10 d$. I don't know 